\title{
Some remarks on stable graphs
}

\section{H.P. Yap}

We introduce some methods of constructing stable graphs and characterize a few classes of stable graphs. We also give a counter example to disprove Holton's conjecture.

\section{Introduction}

A graph $G$ is a strict graph in the sense of Tutte. Let $G_{v_{1} v_{2} \ldots v_{k}}$ be the graph obtained by removing the vertices $v_{1}, v_{2}, \ldots, v_{k}$ and all the edges incident with these vertices, from $G$. Let $A(G)$ be the automorphism group of $G$ and $A(G) v_{1} v_{2} \ldots v_{k}$ be the stabilizer of $\left\{v_{1}, v_{2}, \ldots, v_{k}\right\}$ such that each element in $A(G)_{v_{1}} v_{2} \ldots v_{k}$ fixes $v_{i}$ individually for all $i=1,2, \ldots, k$. Let $|V(G)|$ be the cardinality of the vertex set $V(G)$ of $G$. If there exists a sequence $S=\left\{v_{1}, v_{2}, \ldots, v_{n}\right\}, n=|V(G)|$, of distinct vertices of $G$ such that $A\left(G_{v_{1} v_{2} \ldots v_{k}}\right)=A\left(G v_{v_{1} v_{2} \ldots v_{k}}\right.$ for each $k=1,2, \ldots, n$, then $G$ is said to be stable (otherwise unstable - perhaps an alternative term is recommended as some other writers have used this term in a different sense) and $S$ is called a stabilising sequence of $G$.

In [1], Holton proves, among other results, that

(1) if $G_{v}$ is stable for some $v \in A(G)$, and $A\left(G_{v}\right)=A(G)_{v}$, then $G$ is stable;

(2) the union of $m$ graphs $G_{i}$ is stable if and only if each Received 4 January 1974. 


$$
G_{i} \text { is stable; }
$$

(3) if $A(G)$ is a subgroup of the dihedral group $D_{n}$, $n=|V(G)| \geq 5$, then $G$ is unstable.

We now extend some of Holton's results.

\section{Construction problems}

It is well known that the complement $\bar{G}$ of a disconnected graph $G$ is connected. Also, it is clear that $G$ is stable if and only if $\bar{G}$ is stable. Hence, we can restrict ourselves to the construction of connected stable graphs.

Let $G$ be a stable graph (connected or disconnected) with stabilising sequence $\left\{v_{1}, v_{2}, \ldots, v_{n}\right\}$. Let $v_{1}, v_{2}, \ldots, v_{r}$ be the orbits of $V(G)$ under $A(G)$. We define $G^{*}$ to be the graph obtained from $G$ by adding $a$ new vertex $v_{0}$ to $G$ and adding edges joining $v_{0}$ to all the vertices in $v_{i}$ for some $i$ (one or more) or all $i=1,2, \ldots, r$.

THEOREM 1. G* is stable.

Proof. We shall prove that $\left\{v_{0}, v_{1}, \ldots, v_{n}\right\}$ is a stabilising sequence of $G^{*}$.

We know from the definition that $G_{v_{0}}^{*}=G$. Hence $A\left(G_{v_{0}}^{*}\right)=A(G)$.

It is clear that $A\left(G^{*}\right)_{v_{0}} \leq A(G)$.

We now prove that $A(G) \leq A\left(G^{*}\right) v_{0}$.

Suppose $\phi \in A(G)$. We define a mapping $\phi^{*}$ of $V\left(G^{*}\right)$ onto $V\left(G^{*}\right)$ as follows:

$$
v_{0} \phi^{*}=v_{0}, \quad v_{i} \phi^{*}=v_{i} \phi \text { for every } i=1,2, \ldots, n .
$$

Then $\left(v_{i}, v_{j}\right) \in E\left(G^{*}\right)\left(\left(v_{i}, v_{j}\right)\right.$ is an edge of $\left.G^{*}\right), i, j \neq 0$, implies that $\left(v_{i}, v_{j}\right) \phi^{*}=\left(v_{i} \phi, v_{j} \phi\right) \in E\left(G^{*}\right)$ and $\left(v_{i}, v_{0}\right) \in E\left(G^{*}\right)$ implies that $\left(v_{i}, v_{0}\right) \phi^{*}=\left(v_{i} \phi, v_{0}\right) \in E\left(G^{*}\right)$ because $v_{i}, v_{i} \phi$ belong to the same orbit 
$v_{j}$ for some $j$. Hence $\phi^{*} \in A\left(G^{*} v_{0}\right.$. If we identify $\phi$ with $\phi^{*}$, then $A(G) \leq A\left(G^{*}\right)_{v_{0}}$ and so $A\left(G_{v_{0}}^{*}\right)=A\left(G^{*}\right) v_{0}$. The rest of the proof is immediate.

As a special case of Theorem 1, we have

COROLLARY. If $G$ is a stable graph and $G^{*}$ is the graph obtained from $G$ by adding a new vertex $v_{0}$ to $G$ and adding all the edges joining $v_{0}$ to each vertex of $G$, then $G^{*}$ is stable.

Let $H$ be an induced subgraph of $G$. Let $v_{i} \in V(H)$; we define

$$
D_{1}\left(v_{i}, H\right)=\left\{v_{j} \in V(H) ;\left(v_{i}, v_{j}\right) \in E(H)\right\} \text {. }
$$

Let $H$ and $K$ be two connected stable graphs. Let $\left\{u_{1}, u_{2}, \ldots, u_{m}\right\}$ be a stabilising sequence of $H,\left\{v_{1}, v_{2}, \ldots, v_{n}\right\}$ be

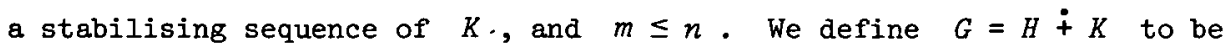
the graph obtained from $H$ and $K$ by identifying $u_{1}$ with $v_{1}$ and putting the two graphs $H$ and $K$ side by side. In other words, $G$ is obtained from the union of $H$ and $K$ by identifying $u_{1}$ with $v_{1}$.

THEOREM 2. Let $G=H \dot{+}$. Suppose $K_{v_{1}}$ is connected. If $H_{u_{1}}$ is not isomorphic with $K_{v_{1}}$ or if $H_{u_{1}}$ is isomorphic with $K_{v_{1}}$ such that

$$
D_{1}\left(u_{1}, H\right) \phi=D_{1}\left(v_{1}, K\right)
$$

for every isomorphism $\phi$ of $H_{u_{1}}$ to $K_{v_{1}}$, then $\left\{u_{1}, u_{2}, \ldots, u_{m}, v_{2}, v_{3}, \ldots, v_{n}\right\}$ is a stabilising sequence of $G$.

Proof. $G_{u_{1}}=H_{u_{1}} \cup K_{v_{1}}$, union of the two disjoint induced subgraphs $H_{u_{1}}$ and $K_{v_{1}}$ of $G$.

If $H_{u_{1}}$ is not isomorphic with $K_{v_{1}}$ then, since $K_{v_{1}}$ is connected, 


$$
A\left(G_{u_{1}}\right)=A\left(H_{u_{1}}\right) \times A\left(K_{v_{1}}\right)
$$

the direct product of $A\left(H_{u_{1}}\right)$ and $A\left(K_{v_{1}}\right)$. Hence

$$
A\left(G_{u_{1}}\right)=A(G)_{u_{1}}
$$

If $H_{u_{1}}$ is isomorphic with $K_{v_{1}}$ such that

$$
D_{1}\left(u_{1}, H\right) \phi=\left\{u_{i} \phi ; u_{i} \in D_{1}\left(u_{1}, H\right)\right\}=D_{1}\left(v_{1}, K\right)
$$

for every isomorphism $\phi$ of $H_{u_{1}}$ to $K_{v_{1}}$, then

$$
A\left(G_{u_{1}}\right)=A\left(H_{u_{1}}\right) \sim S_{2}
$$

the wreath product of $A\left(H_{u_{1}}\right)$ and $S_{2}$, symmetric group of $\{1,2\}$.

Hence $A\left(G_{u_{1}}\right)=A(G)_{u_{1}}$.

The rest of the proof is clear.

REMARKS. If $m>n$, but none of $A\left(H u_{1} u_{2} \ldots u_{k}\right), k=1,2, \ldots, m$, is isomorphic with $A\left(K_{v_{1}}\right)$, then by similar methods, we can show that $G=H+K$ is stable.

It is not difficult to see that the complete bipartite graphs $K_{m, n}$ and in particular, the star graphs $K_{1, t}$ are stable. Hence, we have, with appropriate order of composition and a few restrictions, the following corollaries to Theorem 2.

COROLLARY 1. $K_{m, n}+K_{r, s}$ is stable.

COROLLARY 2. $K_{m, n}+K_{r}$ is stable.

COROLLARY 3. $K_{m}+K_{n}$ is stabze.

Let $G$ be a connected, stable graph with stabilising sequence 
$\left\{u_{1}, u_{2}, \ldots, u_{m}\right\}$. We define $G^{\prime}=G+K_{2}$ to be the graph obtained from $G$ and $K_{2}$ by putting $G$ and $K_{2}$ side by side and adding a new edge $E$ joining $u_{1}$ to a vertex $v_{1}$ of $K_{2}$.

THEOREM 3. If $u_{1} \phi=u_{1}$ for each $\phi \in A(G)$ or there are no monovalent vertices in $G$ then $G^{\prime}$ is stable.

Proof. Suppose $V\left(K_{2}\right)=\left\{v_{1}, v_{2}\right\}$.

If $u_{1} \phi=u_{1}$ for each $\phi \in A(G)$, we can verify that $\left\{v_{1}, v_{2}, u_{1}, u_{2}, \ldots, u_{m}\right\}$ is a stabilising sequence of $G^{\prime}$.

If there are no monovalent vertices in $G$, we can verify that $\left\{v_{2}, u_{1}, v_{1}, u_{2}, \ldots, u_{m}\right\}$ is a stabilising sequence of $G^{\prime}$.

Applying Theorems 1, 2, and 3, together with Holton's result (2), we can construct all stable graphs with $3,4,5$ and 6 vertices from the basic graph $K_{2}$. It is unknown to the author whether we may or may not be able to obtain all the stable graphs $G$ with $|V(G)| \geq 7$ by applying only these methods and accepting that $K_{1, t}$ is stable.

\section{Characterization problems}

Let $G$ be a stable graph with stabilising sequence $\left\{v_{1}, v_{2}, \ldots, v_{n}\right\}$. Suppose $\phi \in A(G)$. Then $\left\{v_{1} \phi, v_{2} \phi, \ldots, v_{n} \phi\right\}$ is also a stabilising sequence of $G$. It would be interesting to investigate the role that stabilising sequences will play in the characterization problems of stable graphs. For instance, it is not difficult to show that if $G$ is connected and stable, then any sequence of the vertex set of $G$ is a stabilising sequence of $G$ if and only if $G$ is the complete graph. We now use this fact to prove

THEOREM 4. Let $G$ be connected and stable. If $\left\{v_{1}, v_{2 \alpha}, v_{3 \alpha}, \ldots, v_{n \alpha}\right\}$ is a stabilising sequence for each permutation a of $\{2,3, \ldots, n\}$, then $G$ is either $K_{n}$ or $K_{1, n-1}$.

Proof. If $G_{v_{1}}$ is connected, then by the previous remark, 
$G_{v_{1}}=K_{n-1}$ and so $G=K_{n}$.

Suppose $G_{v_{1}}$ is disconnected. Let

$$
G_{v_{1}}=H_{1} \cup H_{2} \cup \ldots \cup H_{r} \text {, }
$$

where each $B_{i}$ is a connected component of $G_{v_{1}}$. We can verify without much difficulty that $\left|V\left(H_{j}\right)\right| \geq 2$ for some $j$ is impossible. Hence $G_{v_{1}}$ is the trivial graph with $n-1$ vertices and 0 edges. Hence $A\left(G_{v_{1}}\right)=S_{n-1}$ and this implies that $G=K_{1, n-1}$.

The following is another characterization with respect to the automorphism group.

THEOREM 5. Let $F$ be a group such that for every nontrivial subgroup $F_{1}$ of $F$, any graph whose automorphism group is isomorphic with $F_{1}$ has vertex number greater than the order of $F_{1}$, then any graph $G$ with $A(G)=F$ is unstable.

Proof. The smallest order of $F$ with the above property is 3 . The only group whose order is 3 is the cyclic group $C_{3}$. Any graph $G$ with $A(G)=C_{3}$ has vertex number greater than 3 and $G$ is easily seen to be unstable.

Let $F$ be any group with order greater than 3 and $G$ be any graph with $A(G)=F$. Then for any vertex $v$ of $G, A\left(G_{v}\right)=F_{1}$ is a subgroup of $F$. If $F_{1}$ is the identity group, then by Holton's result (3), $G_{v}$ is unstable. If $F_{1}$ is nontrivial, then by induction hypothesis, $G_{v}$ is unstable and so $G$ is unstable.

COROLLARY. If $A(G)=C_{n}$, the cyclic group of order $n$, and $n$ is 
odd, then $G$ is unstable.

\section{A counter example to Holton's conjecture}

In [1], Holton conjectured that if two graphs $G_{1}$ and $G_{2}$ are such that $A\left(G_{1}\right)=A\left(G_{2}\right)$ where all $G_{1}, \bar{G}_{1}, G_{2}, \bar{G}_{2}$ are connected, then $G_{1}$ is stable if and only if $G_{2}$ is stable.

We now give a counter example to show that Holton's conjecture is not true. The two graphs $G_{1}, G_{2}$ given below satisfy all the conditions in Holton's conjecture. But $G_{1}$ is unstable whereas $G_{2}$ is stable.
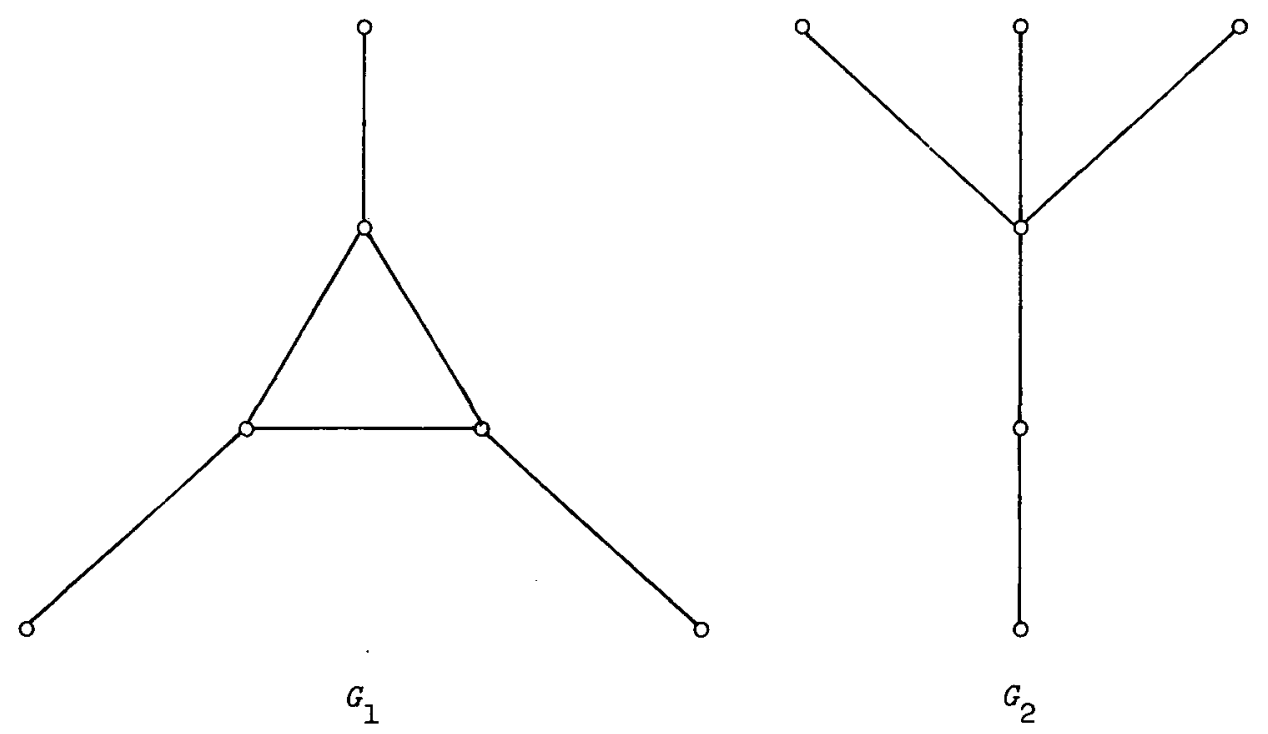

Reference

[1] D.A. Holton, "A report on stable graphs", J. Austral. Math. Soc. 15 (1973), 163-171.

Department of Mathematics, University of Singapore, Singapore.
Department of Combinatorics and Optimization, University of Waterloo, Waterloo, Ontario, Canada. 